\title{
Fundamentals of alveolar diffusion: impact of microscopic solid particles
}

\author{
V. Kulish \\ School of Mechanical and Production Engineering, \\ Nanyang Technological University, Singapore
}

\begin{abstract}
It is demonstrated that diffusion becomes the dominating factor in lower branches of the respiratory duct of humans. A novel mathematical model, based on transport through porous media, for simulating numerically the spatial and temporal gas diffusion process within the alveolar region of the lung is discussed. The model depends on a representative physical property of the alveolar region termed effective diffusivity, function of the diffusivity, solubility, morphology and interface topography of each alveolar constituent. Unfortunately, the direct determination of the effective diffusivity of the alveolar region is impractical because of the difficulty in describing the internal geometry of each alveolar constituent. However, the transient solution of the macroscopic model can be used in conjunction with the lung diffusing capacity (measured in a clinical setting) to determine the effective diffusivity of the alveolar region. With the effective diffusivity known, the three-dimensional effects of red blood cell distribution on the lung diffusing capacity can be predicted via numerical simulations. The results, obtained for normal (random), uniform, centre-cluster, comer-cluster, and several chain-like distributions, unveil a strong relationship between the type of red cell distribution and the lung diffusing capacity. Further, it has been investigated what effect on breathing performance is produced by the presence of passive particles in the alveolar region.
\end{abstract}

\section{Introduction}

Lung diffusion capacity is the lung capacity to exchange oxygen and carbon dioxide. The search for a universal correlation between the lung diffusing capacity and the physical properties of each individual lung alveolar constituent has been a major research trust [1]-[6]. Existing theories for estimating the lung 
diffusing capacity are limited by the difficulty in characterising the incredibly complex internal geometry of the alveolar region, including the distribution of red blood cells within the capillary bed [7].

The lung diffusing capacity is a lumped parameter that can be estimated from relatively simple measurements $([8]-[10])$ and used to indicate abnormalities in the respiratory diffusion process. However, it is difficult to infer from variations in the lung diffusing capacity the precise cause of the abnormality because the sensitivity of the lung diffusing capacity to local changes in the morphology and functionality of the lung is not well known. There is a need for linking the local diffusion process occurring within the alveolar region to the measurable variations in the global lung diffusing capacity parameter.

These observations provide grounds for seeking the development of suitable models that simulate locally the gas diffusion process inside the alveolar region of the lung. Using the classical gas diffusion equation at the alveolus-erythrocyte (microscopic) level within a lung has two major disadvantages:

(1) The dimensional scale of the domain ranges from decimetres to microns, requiring a tremendous numerical resolution.

(2) The complex internal alveolar structure (topology of each constituent) is extremely difficult to access and to map.

This article is a further study of the novel macroscopic model [11] for simulating the gas diffusion within the alveolar region of the lung. This model intends to overcome the scale and structure difficulties, and can be used for preliminary simulations of steady processes. A by-product of this model is the introduction of a macroscopic transport property of the alveolar region, called effective diffusivity. As defined, this property depends on the internal structure of the alveolar region, bringing back the very same structure-related problem the model originally intended to overcome.

\section{Mathematical modelling}

In the human respiratory system, air flows through the nose and/or mouth through the larynx where the epiglottis valve directs the air towards the trachea. The trachea presents a smooth wall covered with ciliary cells that operate like brushes moving the mucus secreted by other cells up the respiratory tract to be expelled via nose/mouth (coughing) or via digestive system (swallowing). It is also along the trachea that temperature and humidity of the incoming air are equilibrated with the body condition.

The incoming air keeps moving down through the trachea, which eventually splits into two bronchial tubes (the primary bronchi, generation $z=1$, shown in Fig. 1), one for each lung. The bronchial tubes are highly elastic and are surrounded by smooth muscle to allow for constriction and dilatation.

The bronchial tubes undergo a series of splits into smaller tubes as the air enters deeper into each lung. The first of these splits is to divide air to each lung lobe through the secondary bronchi. When the bronchial tubes attain diameters smaller than one millimetre, they are then called bronchioles $(\mathrm{BL}$, generation $z=$ 4, Fig. 1). The terminal bronchioles (TBL, generation $z=16$ ) limit the 
conductive zone (no gas exchange) of the respiratory tract. The conductive zone is followed by a transition zone with respiratory bronchioles (from generation $z=$ 17 to $z=19$ ) and a respiratory zone (from generation $z=20$ to $z=23$ ) with alveolar ducts and alveolar sacs. It is in the respiratory zone, where most of the gas exchange takes place.

Observe that the number of bifurcations and the reduced cross-section of the flow passage hinder convection as a gas transport mechanism at the alveolar level. It can be shown that the predominant gas transport mechanism in the alveolar region is by diffusion.

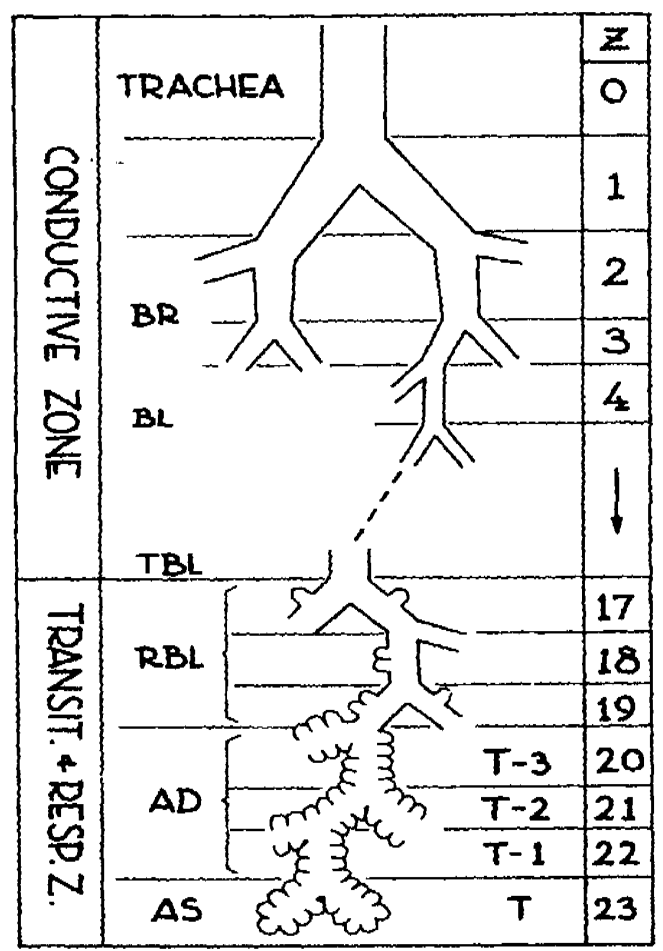

Figure 1: Schematic representation of the respiratory duct of humans (Shields).

\subsection{Diffusion dominance in the alveolar region}

In fact, the effective diameter of the air-ducts can be estimated as $d_{z}=d_{0} 2^{-z / 3}$, where a characteristic $d_{0}$ (diameter of the trachea) is equal to $1.8 \mathrm{~cm}$. The mass conservation requirement, written for different bifurcations (branches) of the respiratory duct, becomes

$$
U_{z} A_{z} N(z)=U_{0} A_{0}=\dot{V}
$$


where $U_{z}$ is the average velocity in $z$-th tube; $N(z)=2^{z}$ is the number of branches at $z$-th generation; $A_{z}=\left(\pi d_{z}^{2}\right) / 4$ is the cross-section area of a single branch; and $\dot{V}$ is the volumetric gas uptake in $\mathrm{m}^{3} / \mathrm{s}$. Note that the power law for the average velocity follows from the mass conservation:

$$
U_{z}=U_{0} \times 2^{-z / 3}
$$

As for the volumetric gas uptake, its value, according to physiological data, equals $3000 \mathrm{ml} / \mathrm{min}$. On average, there are 12 breathing cycles per min. A single cycle consists of the inhalation phase (duration $2 \mathrm{~s}$ ) and the exhalation phase (duration $3 \mathrm{~s}$ ). Therefore, about $24 \mathrm{~s} / \mathrm{min}$ are spent for gas uptake. Thus, under the normal breathing condition (the lowest metabolism), the volumetric gas uptake can be estimated as $\dot{V}=1.25 \times 10^{-4} \mathrm{~m}^{3} / \mathrm{s}$.

The local Reynolds number as well follows the power law, namely

$$
\operatorname{Re}_{z}=\operatorname{Re}_{0} \times 2^{-2 z / 3} \text {. }
$$

Now, from the point of view of mass transport, the distance from the origin of motion, in the case of diffusion transport, is

$$
\delta \sim\left(D_{e f f} t\right)^{t / 2},
$$

where $D_{e f f}$ is the effective diffusion coefficient of the domain in question and $t$ is the time scale of the transport process. In the case of transport by convection, however, the same distance must be estimated as

$$
\delta \sim U t
$$

where $U$ is the average velocity of motion.

Consequently, in terms of the time required for each of these two possible transport processes, one can write:

and

$$
t_{\text {diff }} \sim \delta^{2} / D_{\text {eff }}
$$

$$
t_{\text {conv }} \sim \delta / U
$$

That one of the processes is chosen, for which the time required to deliver mass to the same distance is shorter (the least action). Therefore, from $t_{d i f f}=t_{\text {conv }}$, it follows that

$$
\operatorname{Re}^{*}=\frac{\delta U}{D_{e f f}}=1
$$


It is clear that if the local Reynolds number is smaller than unity, i.e., $\operatorname{Re}<\operatorname{Re}^{*}=1$, then diffusion dominates (almost no flow/convection is possible below this scale). When, however, the local Reynolds number is larger than unity, i.e., $\operatorname{Re}>\operatorname{Re}^{*}=1$, diffusion becomes ineffective as a transport mechanism and convection fully dominates.

Requiring $\operatorname{Re}_{z}=1$ in equation (3), it becomes possible to estimate the generation number, starting from which convection becomes less effective and diffusion grows the dominant transport process. Then,

$$
z^{*}=\frac{3}{2 \ln 2} \ln \operatorname{Re}_{0} \approx 2.164 \times \ln \operatorname{Re}_{0} .
$$

For the critical value of the Reynolds number (transition to the turbulent flow in a pipe), $\operatorname{Re}_{0}=\operatorname{Re}_{c r}=2300$, for instance, $z^{*}=16.75 \approx 17$. That is, at least 17 bifurcations are required in order to kill turbulence at the trachea and make diffusion dominant below $z^{*}$ generations of bifurcating respiratory ducts.

The simple scale analysis presented above amazingly well corresponds to physiological data (see Fig. 1 - the respiratory sacs begin to appear at exactly the seventeenth generation).

\subsection{Macroscopic diffusion equation}

It has been shown in [11] that the following macroscopic transient diffusion equation can be used in order to describe the process of alveolar gas diffusion:

$$
\frac{\partial\langle P\rangle}{\partial t}=D_{e f f} \nabla^{2}\langle P\rangle
$$

where $\langle P\rangle$ is the average partial pressure of the diffusing gas (e.g., $\mathrm{CO}, \mathrm{O}_{2}$ ).

The equivalent effective diffusivity of the alveolar region, $D_{\text {eff }}$ can be determined by simulating numerically the single-breath CO-diffusion procedure done in the laboratory and by comparing the lung diffusing capacity obtained from the numerical results to the lung diffusing capacity obtained experimentally. Once determined, the effective diffusivity can be used with the threedimensional, transient macroscopic diffusion model to investigate the effects of red cell distribution on the lung diffusing capacity.

It has to be noted that the difference between the effective diffusion coefficient, $D_{\text {eff }}$, and the lung diffusing capacity, $D_{L}$, has to be clearly understood. The former is a physical property of the domain (alveolar region) and is independent of boundary conditions. The latter, on the contrary, strongly depends on the boundary conditions and is not a physical property of the domain. This can be simply illustrated as follows.

By separating the time variable in the macroscopic diffusion equation (10), this equation reduces to the ordinary differential equation for the transient function $G$, 


$$
\frac{d G}{d t}+K D_{e f f} G=0
$$

and the Helmholtz equation for the spatial function $F$,

$$
\nabla^{2} F+K F=0
$$

where $K$ is the separation constant and the functions $G$ and $F$ are such that

$$
\langle P(x, y, z, t\rangle=G(t) F(x, y, z) .
$$

The separation constant, $K$, can be found by satisfying the boundary conditions imposed on the Helmholtz equation and, hence, is dependent on those conditions. The general solution of equation (11) is

$$
G(t)=G(0) \exp \left(-D_{L} t\right)
$$

where $D_{L}=K D_{\text {eff }}$ represents the diffusing capacity. It is clear that, although directly proportional to the effective diffusion coefficient, the diffusing capacity depends on the boundary conditions of the problem, because $K$ is the boundary conditions dependent.

\subsection{Lung diffusing capacity and single breath tests}

The lung diffusing capacity obtained in laboratory results from unsteady processes, such as the diffusion process during the single-breath technique [12]. This technique consists of having a subject inhaling a gas mixture with a low concentration of $\mathrm{CO}$, and holding it in for a certain period of time. During this time, $\mathrm{CO}$ will diffuse from the gas region of the alveolus to the RBC's. The process is unsteady because the potential gradient driving the diffusion varies (decays) in time.

The lung diffusing capacity of the alveolar region can be obtained from the single-breath technique results using the Krogh equation ([12], p. 351),

$$
\langle P\rangle_{\nu}(t)=\langle P\rangle_{v_{0}} \exp \left[-\frac{D_{L} P_{r e f}}{V_{A}} t\right],
$$

where $\langle P\rangle_{v_{0}}$ is the initial value of the volume-averaged partial-pressure, the reference pressure $P_{\text {ref }}$ is chosen as the total pressure of dry gases (equal to $9.51 \times 10^{4} \mathrm{~Pa}$, or $713 \mathrm{~mm} \mathrm{Hg}$ ), and $V_{A}$ is the alveolar volume equal to the inspired volume plus the residual lung volume (a representative, normal, value for $V_{A}$ is $4,930 \mathrm{ml}$ STPD).

The characteristic time constant for the diffusion process, according to eqn. (15), is $V_{A} /\left(D_{L} P_{r e f}\right)$, typically equal to $24.4 \mathrm{~s}$ (using $V_{A}=4,930 \mathrm{ml}, D_{L}=17$ $\mathrm{ml}_{\mathrm{Co}} / \mathrm{mmHg} \mathrm{min}$, and $P_{\text {ref }}=713 \mathrm{mmHg}$ ). Notice that using the diffusion and 
solubility laws ([12], p. 350), it is possible to show that the lung diffusing capacity of oxygen is about 1.23 times the diffusing capacity of carbon monoxide. Therefore, the corresponding characteristic time for diffusion of oxygen is approximately equal to 20 seconds.

Note that the solution of eqn. (15) leads to an exponential decay of $\langle P\rangle_{v}$ with time. The lung diffusing capacity can be obtained easily from the partialpressure results by rearranging eqn. (15),

$$
D_{L}=\frac{V_{A}}{P_{\text {ref }} t} \ln \left[\frac{\langle P\rangle_{v}(0)}{\langle P\rangle_{v}(t)}\right]
$$

During clinical single-breath tests, eqn. (16) is used for determining the lung diffusing capacity by measuring $t$ and $\langle P\rangle_{v}(t)$.

To use the single-breath test results it is necessary, then, to model the diffusion process as occurring during the experimental measurement of $D_{L}$. Unfortunately, this means that the transient term of the macroscopic diffusion model must be retained. The model equation becomes then dependent on $D_{e f f}$, a quantity not known a priori.

However, the transient diffusion equation (10) can be used with an estimated $D_{\text {eff }}$ value, in a numerical simulation mimicking the single-breath test. As a first estimate, the $D_{e f f}$ value obtained from the steady-state analysis of Kulish et al. [11] (independent of $D_{e f f}$ ) can be used to determine the time-evolution of the volume-averaged $\mathrm{CO}$ partial pressure. Once the results are obtained, a lung diffusing capacity $D_{L}$ can be found from eqn. (16).

This $D_{L}$ value is not expected to match the lung diffusing capacity found experimentally, because the initial $D_{\text {eff }}$ value (obtained from the steady-state results) was just an approximation to the correct value. Therefore, a new initial estimation of $D_{\text {eff }}$ is used to simulate again the diffusion process. The new results yield a new value of $D_{L}$ to be compared against the experimental value. A predictor-corrector iteration scheme can then be used to fine-tune the $D_{\text {eff }}$ value used in eqn. (10) until the expected (measurable) $D_{L}$ value is obtained from the numerical results. Resulting from this procedure is the correct $D_{e f f}$ value.

\section{Numerical simulation and results}

Three-dimensional, transient numerical simulations of alveolar $\mathrm{CO}$ diffusion, mimicking the single-breath test, is performed by discretising eqn. (10) using finite differences (central-difference), and solving the algebraic equations within a representative alveolar cubic domain for different $\mathrm{RBC}$ distributions, namely: random, uniform, chain, centre- and corner-clustered.

The initial condition for the numerical simulations is $\langle P\rangle(t)=\langle P\rangle_{0}=133.3$ $\mathrm{Pa}$ (= 1 Torr) everywhere within the cubic domain, except at the $\mathrm{RBC}$ locations where the partial pressure of $\mathrm{CO}$ is always equal to zero, i.e., $\langle P\rangle_{\mathrm{h}}=0$. The 
domain boundary is set as impermeable to gas diffusion, therefore, $\partial\langle P\rangle / \partial n=0$ at the boundary, where $n$ is the coordinate along the direction normal to the boundary. The red cell density, defined as the ratio of RBC total volume to the volume of the representative elementary volume, is $\rho=0.034$, a value consistent with 45 percent hematocrit (alveolar region). The cells are distributed randomly within the domain. Additional details of the numerical procedure can be found in [13].

The estimated effective diffusion coefficient found numerically by Kulish et al. [11] from the steady-state simulations, $D_{\text {eff }}=9.4 \times 10^{-8} \mathrm{~m}^{2} / \mathrm{s}$, is used in eqn. (10) as a first guess to simulate the transient process within the alveolar region. After several iterations, it is found that $D_{\text {eff }}=2.68 \times 10^{-7} \mathrm{~m}^{2} / \mathrm{s}$ yields results from which $D_{L}$, obtained from eqn. (16) with $t=10 \mathrm{~s}$, matches the value assumed as representative of random $\mathrm{RBC}$ distribution, $D_{L}=17 \mathrm{ml} \mathrm{Co} /(\mathrm{min} \mathrm{mmHg})$, to within one percent.

First, the time evolution of $\mathrm{CO}$ partial pressure in the course of the singlebreath transient simulation for random, uniform, and clustered RBC distributions was obtained. Next, a more realistic "chain-like" distribution of capillary RBCs was simulated. A chain of RBCs is generated by placing the first RBC somewhere in the discretised domain (at a certain grid-node). A single-file of $\mathrm{RBCs}$ is formed starting from this cell by consecutively following a random path within the cubic domain, from grid-node to grid-node, with each $\mathrm{RBC}$ having at least one neighbouring RBC. The random path of RBCs is continued until the target volume density of RBCs within the domain ( 3.4 percent) is achieved.

The starting point of the chain varies from the centre to the corner of the domain, along one of the diagonals. A half-chain distribution refers to a chain having the first cell placed halfway between the centre and the corner of the domain. A quarter-chain distribution refers to a chain having the first cell placed a quarter of half-diagonal length from the corner of the domain. Corner-chain and centre-chain have their first cell placed at the corner or at the centre of the domain, respectively.

Trying to quantify the location-versus-distance effect of red cell distribution, two geometrical parameters to help characterise each distribution were devised. One of these parameters is the distance between the geometrical centre of the red cell distribution, defined by the co-ordinates $\left(X_{c}, Y_{c}, Z_{c}\right)$, and the centre of the domain at $\left(X_{0}, Y_{0}, Z_{0}\right)$. The co-ordinates of the geometrical centre of the red cell distribution are found from $\left(X_{c}, Y_{c}, Z_{c}\right)=\frac{1}{N} \sum_{i=1}^{N}\left(x_{i}, y_{i}, z_{i}\right)$, where $\left(x_{\mathrm{i}}, y_{\mathrm{i}}, z_{\mathrm{i}}\right)$ are the co-ordinates of each red cell, and $N$ is the total number of red cells in the domain. Hence, the distance to the centre of the domain is simply,

$d=\left[\left(X_{c}-X_{0}\right)^{2}+\left(Z_{c}-Z_{0}\right)^{2}+\left(Z_{c}-Z_{0}\right)^{2}\right]^{1 / 2}$.

The other geometrical parameter characterising the red cell distribution is the effective radius of the red cell distribution, $r=\left(r_{x}^{2}+r_{y}^{2}+r_{z}^{2}\right)^{1 / 2}$, with 
$\left(r_{x}, r_{y}, r_{z}\right)=\frac{1}{N} \sum_{i=1}^{N}\left[\left(X_{c}-x_{i}\right),\left|,\left(Y_{c}-y_{i}\right),\right|, \mid\left(Z_{c}-z_{i}\right)\right]$.

A large $d$-value indicates the cell distribution is far from the centre, hence, close to the boundaries where the diffusion process is less effective. A small $r$ value indicates the red cells are close together, maybe forming a cluster, also leading to a less effective diffusion configuration. Hence, $d$ and $r$ are in principle two good candidates for quantifying the sensibility of the lung diffusing capacity to the three-dimensional red cell distribution.

Values of $d$ and $r$ normalised in respect to the diagonal half-length of the domain, and values of $D_{L}$ for each red cell distribution normalised by the normal $D_{L}$ value are summarised in Table 1 . Observe first that the uniform and centreclustering distributions are not perfectly centred in the domain. This is because the number of red cells necessary to reach 3.4 percent red cell density does not allow a perfectly symmetric distribution in relation to the centre of the threedimensional domain. The distortion caused by this effect, however, is minor.

The random and uniform distributions have very similar $d$ and $r$, and that is why these two distributions yield very similar $D_{L}$. The $d$ and $r$ values for the two cluster distributions indicate that $d$, varying by 270 percent, is not very influential on the value of $D_{L}$, varying only 29 percent.

Based on these observations, the normalised lung diffusion values are plotted versus the normalised radius of distribution, shown in Fig. 2. The continuous line is a polynomial curve-fit, namely

$$
\frac{D_{L}}{D_{L-n}}=-1.6 \frac{r}{s}+19\left(\frac{r}{s}\right)^{2}-68\left(\frac{r}{s}\right)^{3}+93\left(\frac{r}{s}\right)^{4}
$$

Observe that \pm 7.5 percent error bars are also depicted in Fig. 2, a deviation effect attributed to the parameter $d$ not accounted for in eqn. (17).

Further, solid micro-particles were incorporated into the same domain together with the random distribution of RBCs. Those particles were considered as having size of the same order as the size of RBCs. The particles were modelled as an additional (besides RBCs) 'internal' boundary condition such that, for the nodes occupied by them, the mass flux from all (six) neighbouring nodes was kept equal to zero in the course of computations. Obviously, the amount of mass accumulated on the 'particle' nodes was not counted during the averaging procedure.

The numerical experiments were carried on for the values of concentration within a range $0-50$ per cent. The density of randomly distributed RBCs was kept at 0.034 , corresponding to the most realistic case. For each concentration value, the lung diffusing capacity was determined. The result is shown on Fig. 3.

Within the practically interesting range of concentrations of particles, i.e., $0 \leq C \leq 0.5$, the lung diffusing capacity can be quite satisfactorily calculated as the function of the particles concentration by use of a simple formula, namely

$$
D_{L}(C)=D_{L}(0)-10 C \text {. }
$$




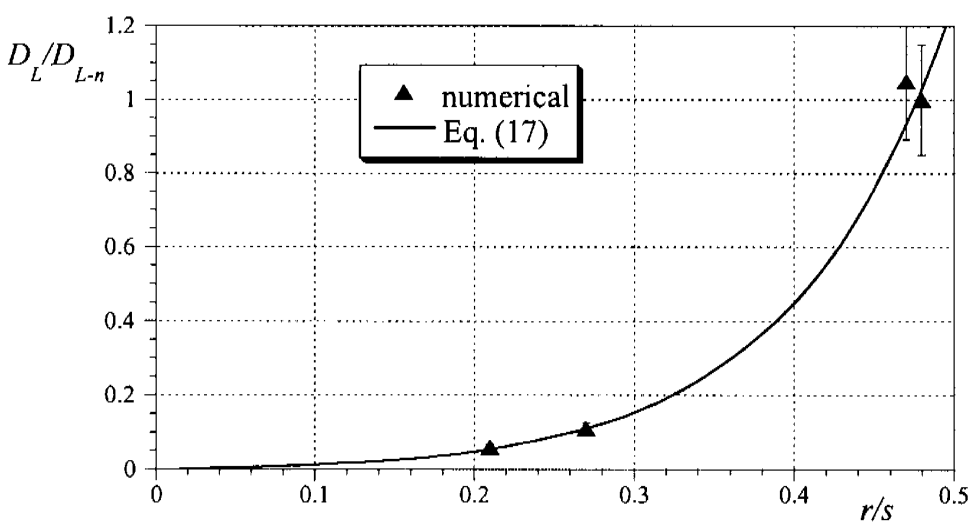

Fig. 2: Normalised lung diffusion coefficient versus normalised distribution radius.

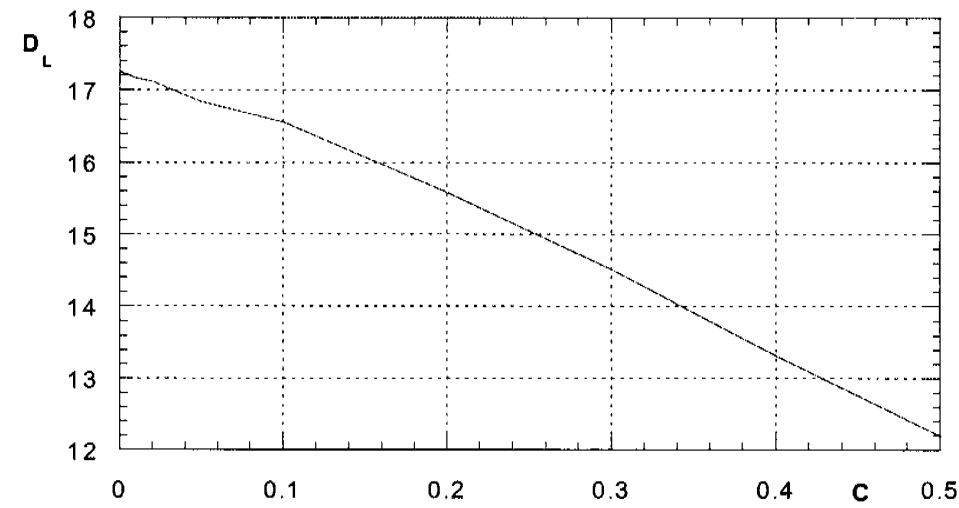

Fig. 3 : Lung diffusing capacity versus concentration of particles.

\section{Conclusions}

The novel macroscopic model of alveolar gas diffusion developed in [11] has now been tested as the transient three-dimensional numerical simulation of the process for different $\mathrm{RBCs}$ distributions. The relationship between the normalised lung diffusing capacity and the parameters characterising an RBC distribution has been established. Provided that the lung diffusing capacity appears to be quite sensitive to the RBC distribution, the hope exists to use this in future for diagnostics of some respiratory diseases.

Another important conclusion, which can be drawn from the present study, is that the lung diffusing capacity is not very sensitive to the presence of solid micro-particles in the alveolar region. 


\section{References}

[1] Roughton, F. J. W. \& Foster, R. E., Relative importance of diffusion and chemical reaction rates in determining the rate of exchange of gases in the human lung, with special reference to true diffusing capacity of the pulmonary membrane and volume of blood in lung capillaries, J. Appl. Physiol., vol. 11, pp. 290-302, 1957.

[2] Weibel, E. R., Morphometric estimation of pulmonary diffusion capacity: Model and method, Respir. Physiol., vol. 11, pp.54-75, 1970.

[3] Crapo, J. D., \& Crapo, R. O., Comparison of total lung diffusion capacity and the membrane component of diffusion capacity as determined by physiologic and morphometric techniques, Respir. Physiol, vol. 51, pp. 183-194, 1983.

[4] Crapo, J. D., Crapo, R. O, Jensen, R. L., Mercer, R. R., \& Weibel, E. R., Evaluation of lung diffusing capacity by physiological and morphometric techniques, J. Appl. Physiol, vol. 64, pp. 2083-2091, 1988.

[5] Fedrespiel, W. J., Pulmonary diffusing capacity: implications of two-phase blood flow in capillaries, Respir. Physiol, vol. 77, pp. 119-134, 1989.

[6] Weibel, E. R., Federspiel, W. J., Fryder-Doffey, F., Hsia, C. C. W., Konig, M., Stalder-Navarro, V. \& Vock, R., Morphometric model or pulmonary diffusing capacity: Membrane diffusing capacity, Respir. Physiol., vol. 93, pp. 125-149, 1993.

[7] Hsia, C. C. W., Chuong, C. J. C., Johnson, R. L., Jr., Critique of conceptual basis of diffusing capacity estimates: a finite element analysis, J. Appl. Physiol, vol. 79, pp 1039-1047, 1995.

[8] Johnson, R. L., Jr., Spicer, W. S., Bishop, J. M. \& Forster, R. E., Pulmonary capillary blood volume, flow, and diffusing capacity during exercise, J. Appl. Physiol., vol. 15, pp. 893-902, 1960.

[9] Newth, C. J. L., Cotton, D. J. \& Nadel, J. A., Pulmonary diffusing capacity measured at multiple intervals during a single exhalation in man, J. Appl. Physiol., vol. 43, pp. 617-625, 1977.

[10] American Thoracic Society, Single breath carbon monoxide diffusing capacity (transfer factor): recommendations for a standard technique, Am. Rev. Respir. Dis., vol. 136, pp. 1299-1307, 1987.

[11] Kulish, V.V., Lage, J.L., Hsia, C.C.W., \& Johnson, R.L., Jr., A Porous Medium Model of Alveolar Gas Diffusion, J. Porous Medium, vol. 2, pp. 263-275, 1999.

[12] Comroe, J. H., Forster, R. E., Dubuis, A. B., Briscoe, W. A. \& Carlsen, E., The lung clinical physiology and pulmonary functions tests, Chicago, Year Book Medical Publisher, pp. 117-121, 1962.

[13] Kulish, V.V. \& Lage, J.L., Fundamentals of alveolar diffusion: a new modelling approach, AUTOMEDICA, vol. 20, pp. 225-268, 2001. 
